\title{
A. FRÖHLICH \\ The Galois module structure of algebraic integer rings in fields with generalised quaternion group
}

Mémoires de la S. M. F., tome 37 (1974), p. 81-86

$<$ http://www.numdam.org/item?id=MSMF_1974_37_81_0>

(C) Mémoires de la S. M. F., 1974, tous droits réservés.

L'accès aux archives de la revue « Mémoires de la S. M. F. » (http://smf. emath.fr/Publications/Memoires/Presentation.html) implique l'accord avec les conditions générales d'utilisation (http://www.numdam.org/conditions). Toute utilisation commerciale ou impression systématique est constitutive d'une infraction pénale. Toute copie ou impression de ce fichier doit contenir la présente mention de copyright.

\section{Numdam}


Journées arithmétiques [1973, Grenoble]

Bull. Soc. math. France,

Mémoire 37,1974, p. 81-86

THE GALOISMODULE STRUCTURE OF ALGEBRAIC INTEGER RINGS

IN FIELDS WITH GENERALISED QUATERNION GROUP

\author{
by \\ A. FRÖHLICH
}

Let $\mathrm{K}$ and $\mathrm{N}$ be algebraic number fields, i.e., extensions of finite degree of the field $Q$ of rational numbers, with $N$ a normal extension of $K$ with Galois group $\operatorname{Gal}(\mathrm{N} / \mathrm{K})=\Gamma$. Let $\theta$ and $\sigma$ be the rings of algebraic integers in $\mathrm{K}$, and in $\mathrm{N}$ respectively. Then $\sigma$ is a module over the group ring $\theta(\Gamma)$, and we are interested in the global structure of this module. One knows (Theorem of Emmy Nother) that $\sigma$ is locally free over $\theta(\Gamma)$ (hence locally free of rank 1 ), if and only if $\mathrm{N} / \mathrm{K}$ is at most tamely ramified. We assume this to be the case, so that we have fixed the local structure of 0 over $\theta(\Gamma)$. It is then convenient to introduce the classgroup $a(\theta(\Gamma))$ of $\theta(\Gamma)$. This classifies the locally free rank one $\theta(\Gamma)$-modules to within stable isomorphism. Here two such modules $M$ and $M^{1}$ are stably isomorphic, if there is a free $\theta(\Gamma)$-module $F$ of finite rank, so that $M \oplus F=M^{1} \oplus F$. We denote by [0] the class in $a(\theta(\Gamma))$ of the module 0 . We wish to determine [0] . What is known in this direction so far concerns special cases, although it is possible to define general invariants of an arithmetic nature, which can be used to describe [o], to unify the known results and to get more general theorems. This will be done elsewhere. Here I shall again consider a particular situation which leads to rather interesting results and problems.

Let now $\mathrm{K}=\mathrm{Q}$, i.e., $\theta=\mathrm{Z}$. Write $\mathrm{H}_{4 \mathrm{~m}}$ for the (generalised) quaternion group of order $4 \mathrm{~m}$. We consider tamely ramified extensions $\mathrm{N} / \mathrm{Q}$ with $\operatorname{Gal}(\mathrm{N} / \mathrm{Q})=\mathrm{H}_{8}$. One knows that $\mathrm{a}\left(\mathrm{Z}\left(\mathrm{H}_{8}\right)\right)$ is of order 2 , and in fact there are exactly two isomorphism classes of rank one $\mathrm{Z}\left(\mathrm{H}_{8}\right)$-modules. Martinet (cf. [4]) derived a handy algorithm to find [0], and he computed examples both for $\checkmark$ to be free, and for 0 to be locally free but not free. We now define an invariant $\mathrm{U}_{\mathrm{N}}$ of tamely ramified fields $\mathrm{N}$ with $\operatorname{Gal}(\mathrm{N} / \mathrm{Q})=\mathrm{H}_{8}$, taking values \pm 1 , by observing that we have an isomorphism 


$$
\theta \cdot a\left(Z\left(\mathrm{H}_{8}\right)\right) \cong \pm 1 \text {, }
$$

and setting

$$
\theta([0])=U_{N} \cdot
$$

We next define a second such invariant. First, let more generally $\mathrm{N} / \mathrm{K}$ be a normal extension of algebraic number fields with arbitrary Galois group $\operatorname{Gal}(\mathrm{N} / \mathrm{K})=\Gamma$. Let $\psi$ be any character of $\Gamma$, in the sense of representation theory over the complex numbers. The extended Artin L-series then satisfies a functional equation

$$
\Lambda(s, N / K, \psi)=W(N / K, \psi) \Lambda(1-s, N / K, \bar{\psi})
$$

where $\bar{\psi}$ is the complex conjugate of $\psi$, and where the "root number" $\mathrm{W}(\mathrm{N} / \mathrm{K}, \psi)=\mathrm{W}(\psi)$ has absolute value 1 . If $\psi=\bar{\psi}$ is real valued, then one knows that $\cdot \mathrm{W}(\psi)= \pm 1$.

Now return to the case $\mathrm{K}=\mathrm{Q}, \operatorname{Gal}(\mathrm{N} / \mathrm{Q})=\mathrm{H}_{8}$. All characters of $\mathrm{H}_{8}$ are real valued, and by the multiplicativity of root numbers under character addition, it suffices to consider only irreducible $\psi$. Moreover for real Abelian, i.e., quadratic or trivial characters one knows that the value of the root number is 1 . This just leaves the unique two-dimensional irreducible character $\psi_{8}$ of $\mathrm{H}_{8}$, and we define

$$
\mathrm{W}\left(\mathrm{N} / \mathrm{Q}, \psi_{8}\right)=\mathrm{W}_{\mathrm{N}}
$$

Then I proved (cf. [1]) :

Theorem 1. If $\mathrm{N} / \mathrm{Q}$ is tamely ramified, $\operatorname{Gal}(\mathrm{N} / \mathrm{Q})=\mathrm{H}_{8}$, then $\mathrm{U}_{\mathrm{N}}=\mathrm{W}_{\mathrm{N}}$.

My attack on this problem was encouraged by Serre, who had computed $\mathrm{U}_{\mathrm{N}}$ and $\mathrm{W}_{\mathrm{N}}$ in one case where they both have value -1 , followed by Armitage, who altogether computed twelve examples. I also showed that $\mathrm{W}_{\mathrm{N}}$ takes each of the values \pm 1 infinitely often, even with further arithmetic "boundary conditions" imposed (cf. [1]).

This theorem is rather surprising. The proof is based on a good arithmetic classification of the fields $\mathrm{N}$, which essentially goes back to papers of mine of twenty years ago, but it does not give any real insight into why such a theorem'should hold. Some other alternative proof would therefore be desirable. 
Another problem is that of a possible generalisation of Theorem 1. Before one can formulate a conjecture one has to get good definitions of the invariants $\mathrm{U}_{\mathrm{N}}$ and $\mathrm{W}_{\mathrm{N}}$ and this itself involves serious and interesting problems. I shall here concentrate on $\mathrm{W}_{\mathrm{N}}$.

For the root numbers our original procedure for $\mathrm{H}_{8}$ will not work. We shall call $\psi=\psi_{4 \mathrm{~m}}$ a quaternion character of order $4 \mathrm{~m}$ if it is an irreducible real valued character of $\mathrm{H}_{4 \mathrm{~m}}$ of degree 2 , corresponding to a faithful representation of $\mathrm{H}_{4 \mathrm{~m}}$. There are such characters (for $\mathrm{m}>1$ of course), and, for given $\mathrm{m}$, they are all conjugate over $\mathrm{Q}$. In general one cannot expect that for any two such characters the root numbers coincide. In fact, we have

Theorem 2. There is a unique field $\mathrm{N}$ containing $\mathrm{Q}(\sqrt{5})$ with $\operatorname{Gal}(\mathrm{N} / \mathrm{Q})=\mathrm{H}_{20}$ ' so that $N / Q(\sqrt{ } 5)$ has conductor 55 . There are exactly two quaternion characters $\psi$ and $\psi^{\prime}$ of order 20 , and for this field $N$

$$
W(N / Q, \psi)=-W\left(N / Q, \psi^{\prime}\right) \text {. }
$$

Note however that $\mathrm{N} / \mathrm{Q}$ is wildly ramified. In fact we do get

Theorem 3. Let $\mathrm{N} / \mathrm{K}$ be a normal extension with $\mathrm{Gal}(\mathrm{N} / \mathrm{K})=\mathrm{H}_{4 \mathrm{~m}}$. If $\mathrm{N} / \mathrm{K}$ is tamely ramified, then the values of the root numbers $\mathrm{W}(\mathrm{N} / \mathrm{K}, \psi)$, for all quaternion characters of order $4 \mathrm{~m}$ coincide.

Using this theorem we can now define, for a tamely ramified field N/Q with $\operatorname{Gal}(\mathrm{N} / \mathrm{Q})=\mathrm{H}_{4 \mathrm{~m}}$, the invariant $\mathrm{W}_{\mathrm{N}}$ as the common value of the $\mathrm{W}(\mathrm{N}(\mathrm{Q}, \psi))$, for $\psi$ a quaternion character of order $4 \mathrm{~m}$.

We shall say a few words about the background to Theorems 2 and 3 . If $\operatorname{Gal}(\mathrm{N} / \mathrm{K})=\mathrm{H}_{4 \mathrm{~m}}$ then we have a field tower $\mathrm{K} \subset \mathrm{E} \subset \mathrm{N}$, where $\mathrm{E}$ is quadratic over $\mathrm{K}, \mathrm{N}$ cyclic over $\mathrm{E}$. Let $\phi$ be the idele class character of $\mathrm{N} \quad \mathrm{K}$, for which $\mathrm{E}=\mathrm{K}_{\phi}$ is the class field. Let $X$ be an idele class
character of $\mathrm{E}$ with $\mathrm{N}=\mathrm{E}_{\chi}$. Viewed as a character of Gal(N/E),
this $X$ will induce a quaternion character $\psi$ of order $4 \mathrm{~m}$ of
$\mathrm{E} \quad \mathrm{Gal}(\mathrm{N} / \mathrm{K})$, and all such quaternion characters are given in this manner.
Moreover, we ave $\mathrm{W}(\mathrm{N} / \mathrm{K}, \psi)=\mathrm{W}(\mathrm{X})$, and the various Abelian charac-
ters $X$, with $\mathrm{N}=\mathrm{E}_{\chi}$, are all conjugate over $\mathrm{Q}$. Finally, the fact 
that $X$ induces a quaternion character is expressed exactly in the equation $x \mid C_{K}=\phi$, where $x \mid C_{K}$ is the restriction of $x$ to the idele class group $\mathrm{C}_{\mathrm{K}}$ of $\mathrm{K}$. We thus have to compare root numbers of Abelian characters which are conjugate over $Q$.

Let $Q^{\text {Cyc }}$ be the maximal cyclotomic field inside the field of complex. numbers. The Galois group $\operatorname{Gal}\left(Q^{C Y C} / Q\right.$ ) can be identified with $\prod_{p} U_{p}$ (product over all finite primes), where $U_{p}$ is the group of p-adic units. This Galois group acts in a natural manner both on the Abelian characters and on their root numbers. Namely if $\eta$ is an $r$-th root of unity, and $u n \equiv 1(\bmod r)$, with $n \in Z$, then $\eta^{u}=\eta^{n}$. For $u \in \prod_{p} U_{p}, a \in Q^{*}$ define $\left(\frac{u}{a}\right)$ by $\left(\frac{u}{a}\right)=\prod_{p}\left(\frac{u, a}{p}\right)_{2} \quad$ (product of Hilbert symbols),

or equivalently

$$
\left(\frac{\mathrm{u}}{\mathrm{a}}\right)=\sqrt{\mathrm{a}}^{\mathrm{u}} / \sqrt{\mathrm{a}} .
$$

We then have

Theorem 4. For any Abelian character $X$ of an algebraic number field $E$, and for $u \in \prod_{p} U_{p}=\operatorname{Gal}\left(Q^{C y C} / Q\right)$,

$$
w(x)^{u}=w\left(x^{u}\right) x^{u}(u)\left(\frac{u}{N f(x)}\right)\left(\frac{u}{c(x)}\right)
$$

where $N f(X)$ is the absolute norm of the conductor $f(X)$, and where $c(X)=(-1)^{Y}, \quad Y$ being the number of real places of $E$ at which $X$ is ramified.

Note for the definition of $\chi^{u}(u)$ that $u$ is a rational idele, hence an idele of $E$. The case relevant to us is given by the

Corollary. If $W(X)= \pm 1$ then

$$
w(x) / w\left(x^{u}\right)=x^{u}(u)\left(\frac{u}{N f(x)}\right)\left(\frac{u}{c(x)}\right) .
$$

Serre has pointed out that the formula of Theorem 4 yields a similar formula for non-Abelian characters, namely

$$
\text { (*) } \quad W(\psi)^{u}=W\left(\psi^{u}\right) \delta_{\psi}^{u}(u)\left(\frac{u}{N f(\psi)}\right)\left(\frac{u}{c(\psi)}\right) \text {. }
$$

Here $\delta_{\psi}$ is the "determinant" of $\psi$, i.e. viewed as a character of a Galois 
group it is given by

$$
\delta_{\psi}(\gamma)=\operatorname{det} T(\gamma)
$$

if $Y \rightarrow T(\gamma)$ is a representation corresponding to $\psi$. Also $c(\psi)=\Pi c_{v}(\psi)$, $\mathrm{v}$ running through the real places of the base field $\mathrm{E}$, with $\mathrm{c}_{\mathrm{v}}(\psi)=(-1)^{n_{\mathrm{v}}}$., where $\mathrm{n}_{\mathrm{v}}$ is the number of eigenvalues -1 of the $\mathrm{v}$-Frobenius element $\sigma_{\mathrm{v}}$ in a representation corresponding to $\psi$. In other words $c_{v}(\psi)=\delta_{\psi}\left(\sigma_{v}\right)$.

Note that formula (*) allows one to regain a result of Dwork's, in answer to a question of Hasse, on the field in which $W(\Downarrow)$ lies.

To get Theorem 2 one takes $\mathrm{E}=\mathrm{Q}(\sqrt{5})$, with the appropriate $\mathrm{X}$ of order 10 , ramified at 5 and at 11 . The operator $u$ is then chosen to be $u_{5}=3_{5}, u_{p}=1$ for $p \neq 5$.

Theorem 3 follows from an explicit formula for $W(X)$. Let $n$ be the discriminant of $E / K$ and let $E=K(\Delta), \Delta^{2} \in K$, with $\Delta$ integral and square free at all prime divisors $p$ of $b$ in $E$. The part of $(\Delta)$ "prime to $b "$ is then a fractionnal ideal $a$ in $K$. Let moreover $f^{*}$ be the part of $f(X)$ "prime to $b$ ". $f^{*}$ is an ideal in $K$. One then has

Theorem 5. If $N=E_{X}, E=K_{\phi}$ quadratic over $K$, and if $N / K$ is tamely ramified and Gal. $(\mathrm{N} / \mathrm{K})=\mathrm{H}_{4 \mathrm{~m}}$, then

$$
W(X)=\left(\frac{2}{\mathfrak{b}}\right) \phi\left(f^{*}\right) \underset{\left.\mathfrak{P}\right|_{\mathbb{D}}}{\pi} \mathrm{X}_{\mathfrak{B}}(\Delta)
$$

Theorem 3 follows almost immediately. For, $W(x),\left(\frac{2}{b}\right)$ and $\phi\left(f^{*}\right)$ clearly take only \pm 1 as possible values, hence so does $\prod_{\left.\mathfrak{B}\right|_{\mathbb{D}}} x_{\mathcal{P}}(\Delta)$. Therefore replacing $x$ by $\chi^{\mathrm{u}}$ will not alter anything.

The proofs of Theorems 2-5 are contained in reference [3] below.

$$
-:-:-:-
$$

\section{BIBLIOGRAPHY}

[1] A. FRÖHLICH - Artin root numbers and normal integral bases for Quaternion Fields, Inventions Math. 17, 143-166 (1972). 
[2] A. FRÖHLICH and J. QUEYRUT - On the functionnal equation of the Artin L-function for characters of real representations. To appear in Inventiones Math.

[3] A. FROHLICH - The root numbers, conductors and representations of Artin for generalised quaternion groups. To appear.

[4] J. MARTINET - Modules sur l'algèbre du groupe quaternionen. Ann. Sci. de l'Ecole Normale Sup. 4(3) 399-408 (1971).

$-:-:-:-$
A. FROHLICH
University of London,
King's College,
Strand, LONDON, WC 2 R 2 L S. 